\title{
GLAD!
}

Revue sur le langage, le genre, les sexualités

$07 \mid 2019$

Varia

\section{Frontières de l'humain et technologies de genre monstrueux}

\section{Le Fol marbre de Dennis Cooper}

Human Borders and Monstrous Gender Technologies: Dennis Cooper's The

Marble Swarm

\section{Kevin Lambert}

\section{OpenEdition}

\section{Journals}

Édition électronique

URL : http://journals.openedition.org/glad/1691

DOI : 10.4000/glad.1691

ISSN : 2551-0819

\section{Éditeur}

Association GSL

\section{Référence électronique}

Kevin Lambert, « Frontières de l'humain et technologies de genre monstrueux », GLAD! [En ligne], 07 | 2019, mis en ligne le 05 décembre 2019, consulté le 17 décembre 2020. URL : http:// journals.openedition.org/glad/1691; DOI : https://doi.org/10.4000/glad.1691

Ce document a été généré automatiquement le 17 décembre 2020.

\section{(c) (i) $(9)$}

La revue GLAD! est mise à disposition selon les termes de la Licence Creative Commons Attribution Pas d'Utilisation Commerciale - Pas de Modification 4.0 International. 


\title{
Frontières de l'humain et technologies de genre monstrueux
}

\author{
Le Fol marbre de Dennis Cooper \\ Human Borders and Monstrous Gender Technologies: Dennis Cooper's The \\ Marble Swarm
}

Kevin Lambert

1 The Marbled Swarm est le dernier roman en date de l'américain Dennis Cooper, connu notamment pour le cycle de Georges Miles, pentalogie romanesque publiée de 1989 à 2000. Le roman The Sluts (2005), entièrement écrit à partir de fausses critiques web de garçons escortes, ainsi que le blog qu'il tient depuis $2010^{1}$ en plus de plusieurs pièces de théâtre, montées dès 2004 par la metteure en scène française Gisèle Vienne, ont notamment ouvert l'œuvre de l'écrivain à d'autres formes d'écriture. The Marbled Swarm - Le fol marbre ${ }^{2}$ en français - constitue toutefois une exception dans la production de l'écrivain, reconnu pour son style associé au New Narrative américain et à la Blank Fiction (Patoine 2015 : 54), caractérisé par une écriture sans fioritures, crue, orale, directe et nerveuse. Bien qu'on y rencontre plusieurs éléments qui font la particularité de l'univers de Cooper-un regard amoral ; une homosexualité thématisée comme prédatrice ou masochiste et qui s'accompagne souvent de désirs incestueux et pédophiles; des personnages qui ont soit envie de mourir, soit envie de tuer; une narration métadiscursive ; des descriptions pornographiques; une esthétique gore -, le roman marque un changement dans son œuvre sur le plan linguistique et stylistique. Le fol marbre adopte en effet un style baroque parodiant le ton raffiné des romans gothiques et une écriture surfaite de tournures pompeuses et de paraphrases métaphoriques, qui mélange les registres soutenus et le slang.

2 On pourrait qualifier le narrateur sans nom du Fol marbre avec les mots de Clarice Starling, dans Le Silence des agneaux de Jonathan Demme, lorsqu'elle répond à l'agent qui lui demande si Hannibal Lecter est « some kind of vampire » : "They don't have a name for what he is ", dit-elle. Pas de nom commun pour qualifier celui dont le nom propre est devenu l'expression même de ses crimes abominables; le monstrueux 
atteint parfois les limites du langage, semble nous dire le film. S'il n'y a pas un bon mot pour nommer la monstruosité du personnage principal et narrateur du roman de Cooper, c'est qu'il y en a plusieurs : cannibale, milliardaire, violeur, pédophile, raciste, incestueux, nécrophile, misogyne, meurtrier en série, dangereusement narcissique.

Dans ce texte, je tenterai de montrer que le Fol marbre est un roman qui vise à révéler les mécanismes d'édification de toute norme sexuelle et qui, pour ce faire, questionne le lien entre le genre et le monstre. D'abord le genre (gender) au sens où l'entend la théorie queer depuis Butler, c'est-à-dire compris comme une construction culturelle et discursive historiquement et socialement variable, à laquelle participent plusieurs disciplines, allant des systèmes sémiotiques (comme la littérature) à la recherche scientifique (dont le rôle dans la fabrication des catégories sexuelles a notamment été démontré par Thomas Laqueur [2013], Anne Fausto-Sterling [2012] et Paul B. Preciado [2008]). La production de l'être "genré » est ainsi motivée par de nombreux facteurs variant dans l'espace et le temps: les "catégories de sexe» (Wittig 2013: 37-44) en place, la conception qu'une culture se fait du corps et d'une sexualité «normale», les différentes « orientations sexuelles " qu'elle s'invente pour nommer cette sexualité et l'établir en "identité », etc. Le monstre, ensuite, dont l'aspect ou les comportements sont considérés comme un écart à la norme sociale et qui remet en question, voire rompt avec les frontières classiques posées entre l'intérieur et l'extérieur, la forme et l'informe, l'humain et l'animal, le corps et la machine, l'étranger et le familier, le soi et l'autre, le bien et le mal, le masculin et le féminin, dans une déconstruction faite chair qui suscite des sentiments d'horreur, d'abjection ou de dégoût. Je considère que le roman de Cooper laisse entendre que le monstre, à la manière du genre, est le fruit d'une fabrication culturelle et discursive, fabrication que le texte nous permet de saisir comme telle, en explorant les différents dispositifs sexuels et culturels motivant ces productions; nous verrons que chez Cooper il est produit par les discours, les technologies et les peurs qui croisent le sexe avec l'horreur. Le roman s'inscrit de cette manière dans une longue tradition de littérature "monstrueuse» qui fonde ce personnage sur un écart "horrifique» envers la sexualité hétéronormative et le système de genre reposant sur l'opposition binaire masculin/féminin. Nous verrons cependant que le frisson qu'il provoque ne vise plus, comme au XIX ${ }^{e}$ siècle, à produire un sentiment de terreur homophobe envers tout ce qui échapperait à la "norme", mais que le roman, en déplaçant le point de vue de la représentation du monstre à sa production, inquiète plutôt les processus de sédimentation et d'intelligibilité même du «normal » et de l'« anormal », du " marginal » et du « dominant ». La fiction horrifique de Cooper, en somme, n'identifie plus le trouble dans le genre que produit le monstre à ses aspects les plus terrifiants dans l'objectif de servir les visées sexistes et racistes de certaines idéologies ${ }^{3}$, mais table plutôt sur cet héritage dans l'objectif de déconstruire avec violence les oppositions supposées étanches sur lesquelles se fonde le système sexuel dominant.

Le monstre qui nous raconte Le fol marbre - la liste citée plus tôt le montrait déjà - se construit à l'intersection de caractéristiques économiques, raciales et criminelles, de comportements ou de désirs sexuels frappés d'un interdit social qui servent à composer son abjection fictive. Posant que « les écrivains sont marqués par le discours social de leur temps sur le monstre et la dégénérescence » (Chassay 2014 : 63), j'avancerai que le roman coalise certains changements importants dans l'imaginaire contemporain du monstre, dont l'histoire est esquissée par Jack Halberstam dans Skin Shows: Gothic Horror and the Technology of Monsters. L'ouvrage, qui propose une lecture socio-historique de la 
monstruosité attentive à son rapport au genre, avance que ces personnages avaient, dans la littérature gothique, pour fonction l'identification négative de la société anglaise $\mathrm{du} \mathrm{XIX}^{\mathrm{e}}$ siècle $(S S, 22)$. Le monstre fonctionnait alors, selon l'auteur, à la manière d'un écran sur lequel les peurs de la classe dominante se voyaient projetées : «The monster [...] condenses various racial and sexual threats to nation, capitalism, and the bourgeoisie in one body. If the Gothic novel produc[ed] an easy answer to the question of what threatens national security and prosperity (the monster), the Gothic monster represent[ed] many answers to the question of who must be removed from the community at large » $(S S, 3)$. Cette identification négative, produisant une définition de l'humain par antithèse, aurait contribué selon Halberstam à asseoir la norme bourgeoise, capitaliste, blanche, hétérosexuelle en élaboration et en quête de repères à l'époque victorienne $(S S, 22)$. Les incarnations contemporaines du monstre dans la fiction marqueraient cependant, selon Halberstam, un changement quant à la fonction sociale de la figure, courant dans lequel s'inscrit Le fol marbre. Plutôt que d'unir sous son masque grimaçant différents éléments qui menacent la culture dominante afin de protéger celle-ci en identifiant le monstre comme son envers, son extérieur, son Autre absolu, le monstre contemporain se caractériserait par son rapprochement, sa proximité avec l'humain et avec la norme 4 . Pour le dire avec Halberstam, « horror now disrupts dominant culture's representations of family, heterosexuality, ethnicity, and class politics » (SS, 23).

Le narrateur du Fol marbre, que je propose de lire sous le signe du monstrueux, occupe à mon avis une fonction semblable dans l'imaginaire du monstre : il fonctionne comme un instrument narratif servant à critiquer la culture dominante plutôt qu'à en affermir les assises. Nous verrons que le monstre lui-même ainsi que les actes immondes qu'il commet sont rendus possibles en raison de la marginalité ultra-permissive dans laquelle se trouve ce personnage. Dans le Fol marbre, c'est un dérapage du monde social qui produit le monstrueux, et non l'inverse ; il s'agit du premier changement important dans l'imaginaire littéraire du monstre qui peut être observé chez Cooper. Comme nous le verrons, ce passage du monstre vers le dominant s'accompagne d'actions et de désirs exorbitants, connotés comme abjects dans le récit et qui- c'est le deuxième changement que j'aimerais commenter - visent à ébranler la notion classique du sujet, et par là même un ensemble de binarismes qui fondent son projet. Le monstre contemporain, suggère le roman de Copper, ne pourrait ainsi plus se saisir dans une perspective strictement identitaire puisque la dispersion du sens de l'humain, du genre et du corps qu'il produit ne peut se rassembler sous aucune unité discursive ou narrative, dimensions qui fondent traditionnellement cette notion. Le fol marbre, plutôt que de proposer un modèle stable du monstrueux, lui substitue une conception productive, générative s'apparentant à la conception butlérienne du genre. Le monstre chez Cooper est le fruit d'un processus de fabrication qui l'institue comme tel à la fois dans la diégèse et dans le texte. Par le fait même, le roman menace les limites autrefois stables du monstrueux : si on ne naît pas monstre, on le devient, personne n'est à l'abri de ce devenir-monstre qui hante Le fol marbre. Pas même le lecteur ou la lectrice, comme nous le verrons finalement. Cette dimension que je nomme performative du monstrueux constitue le dernier changement dans la figure contemporaine du monstre que je commenterai. 


\section{Le monstre aux commandes}

6 Le monstre du Fol marbre est paradoxal. Nommons, parmi les contradictions étonnantes qui le caractérisent, sa grande beauté, qu'il ne se gêne d'ailleurs pas pour vanter :

En espérant que ma façon de m'adresser des louanges n'a pas noyé ma véritable perfection dans une surenchère métaphorique, permettez-moi de suggérer qu'en matière de charme je pourrais consacrer de nombreuses pages assommantes et joliment surchargées à expliquer en quoi ma beauté est un fait qu'aucune personne m'ayant rencontré n'a jamais contesté, même si j'imagine qu'une simple vérification fera l'affaire. $(F M, 17)$

7 Contrairement au monstre «traditionnel », celui de Cooper échappe à la logique de la physiognomonie, cette pseudo-science "selon laquelle il est possible d'atteindre les profondeurs de l'intériorité humaine par l'observation d'éléments extérieurs " (Stiénon \& Wicky 2013:5) et qui a longtemps marqué les représentations monstrueuses en établissant une équivalence entre l'aspect physique horrifique et une intériorité tout aussi terrible ${ }^{5}$. Le monstre du Fol marbre est attirant. Pour souligner ce paradoxe, la narration détourne une figure langagière fréquemment employée dans la description du physique monstrueux en littérature, celle de la prétérition, qui sert ici à décrire une beauté si fulgurante que le texte aurait du mal à la rendre. Il faudrait en effet une "surenchère métaphorique " et "de nombreuses pages assommantes et joliment surchargées " pour transmettre toute son ampleur au lecteur ou à la lectrice. Le contreemploi de la prétérition, qui avoue les limites du langage pour évoquer le beau plutôt que le laid, place déjà sur le plan de la monstruosité l'apparence physique sublime du narrateur. Reste toutefois à clarifier cet oxymore : que peut signifier, dans l'imaginaire tératologique, un monstre beau? Deuxième paradoxe, bien que le narrateur monstrueux commette nombre d'actes sanguinaires comme le meurtre, la dévoration de chair humaine ou la volupté avec des cadavres, il n'est pas présenté dans le livre comme un être bestial. Le monstre du Fol marbre n'est pas un ogre infâme vivant dans une caverne, mais un fils de milliardaire cultivé qui connaît - sans l'apprécier - l'art contemporain, et qui a grandi dans un loft parisien « conçu [designed] par l'architecte Philippe Starck, si tant est qu'une étendue vide et rectangulaire stérile subdivisée à faible luminosité puisse être considérée comme une idée [a design]» $(F M, 90)$. Cela nous mène à une autre question : que peut signifier la coexistence chez un même personnage de ces deux dimensions en apparence contradictoires - la bestialité des actions et le raffinement psychologique ? Le premier exemple comme le second montrent que le monstre du Fol marbre tend vers le dominant; le roman de Dennis Cooper nous invite à penser le monstrueux dans son versant riche et puissant, qui ne se distingue plus franchement de la norme, mais qui négocie avec elle une nouvelle relation, en exagérant certains de ses mécanismes. Si le monstre y est beau et cultivé plutôt qu'affreux et sauvage, c'est que le roman marque un changement par rapport à la figure traditionnelle du monstre: il devient, chez Cooper, un dispositif critique des discours sociaux régnants, dont il révèle la logique perverse. Il est d'ailleurs possible de lire les comportements les plus scandaleux du personnage comme un effet de la position privilégiée qu'il occupe dans la société.

8 Soulignons d'abord que la puissance du monstre marque avant tout la narration $d u$ texte. Si le mot «monstre» provient du même étymon que le verbe «montrer» 
(Picoche $2008: 324)$ et qu'en littérature le monstrueux « n'existe qu'à travers le regard de l'autre " (Chassay 2014: 7), chez Dennis Cooper, la narration autodiégétique donne voix au monstre ; c'est lui qui montre. « The beasts who were told but never got to tell » $(S S, 163)$ dans la littérature gothique semblent en effet plus loquaces dans la littérature contemporaine et particulièrement dans ce roman de Cooper qui donne à lire un narrateur conscient de son pouvoir tyrannique sur l'histoire qu'il nous raconte. Il interpelle en effet fréquemment le lecteur ou la lectrice pour anticiper ses réactions, déjouer ses attentes, souligner un brusque changement de sujet ou promettre un développement ultérieur que le texte ne fournit pas toujours. Plus encore, cette emprise que lui confèrent les rênes du récit s'exprime parfois de manière symbolique sous la forme d'une violence faite au lecteur ou à la lectrice : à titre d'exemple, dès les premières pages, le narrateur se décrit comme " un minable [the creep] auquel vous finirez quand même par tendre votre autre joue » $(F M, 14)$. La référence au discours biblique assigne ici au lecteur implicite ${ }^{6}$ une position masochiste par rapport au narrateur, qui le menace tacitement d'une violence (sous-entendue malgré la coupure de la première partie du verset : «On te frappe sur la joue droite? Présente l'autre » [La Bible $2001: 2225]$ ) tout en déterminant déjà sa réaction de soumission. Plus loin, on rencontre une autre forme de menace en réponse aux hypothèses que le narrateur prête au lecteur implicite: «D'après ce que vous avez lu jusque-là, vous devez penser que le chien de Pavlov aurait pu répondre à ma place. Votre hypothèse est juste, toutefois je vous rappelle que de tirer des conclusions viles ne sera bientôt plus à votre avantage » $(F M, 46)$. Le protagoniste exprime par ces mots le regard qu'il imagine être porté sur ses actions, répondant à l'opinion fantasmée du lecteur implicite par une menace qui demeure imprécise, et ce, tout en attisant simultanément son intérêt, laissant planer un « bientôt » qui annonce un développement à venir.

9 La position dominante inférée pour le narrateur par le texte se révèle de manière encore plus évidente dans la description du «fol marbre» qui donne son titre au roman. Nom donné à une forme langagière sophistiquée forgée par le père du narrateur, le "fol marbre » posséderait, selon ce dernier, un caractère presque fantastique, puisqu'il serait muni d'un pouvoir hypnotique sur ses allocutaires. Mimant la logique capitaliste par l'accumulation de richesses qu'il aurait permis au père du narrateur, mais aussi par sa faculté à piller les autres langues occidentales de leurs «meilleurs hits linguistiques" $(F M, 188)$ afin de les incorporer à sa propre voix - c'est sa dimension "marbrée »-, le fol marbre absorbe tout et se " parle à un rythme ardu enchaînant des séries de phrases poisseuses [at a taxing pace in trains of sticky sentences] qui rassemblent les pensées avec autant de généralité qu'un [as broadly as] aspirateur » $(F M, 67)$. Le «swarm » qui forme le terme original (marbled swarm) renvoie précisément à cette dernière dimension du fol marbre, soutenue par la comparaison avec l'aspirateur: ses vertus reposeraient sur les multiples interprétations qu'il peut susciter, sur la dispersion du sens à la manière d'un essaim qu'il produit plutôt que sur sa clarté. La confusion qu'il provoque, la dissémination qu'il induit, font du fol marbre « une manière de parler [...] [qui] rend le discours d'une personne aussi ouvert [openended] que l'air qu'il remplit et pourtant aussi dangereux à parcourir qu'une pièce plongée dans le noir et encombrée dans laquelle on a caché, disons, un milliard d'euros » $(F M, 67)$.

10 Ouvrons une parenthèse pour mentionner que la référence à ce "milliard d'euros " n'est pas anodine, puisque «le charme apparent [de ce style verbal] a fait ses preuves en accumulant [1]es milliards [du père], désormais les miens » $(F M, 66)$, comme nous 
l'apprend le narrateur. L'ajout, suivant la virgule, de « désormais les miens » à la fin de cette phrase vient reproduire le mouvement de la passation de la fortune d'une génération à l'autre, plaçant le narrateur comme héritier. Ce dernier, qui reçoit après la mort de son père cette fortune immense, correspond ainsi à une figure présente dans l'imaginaire social contemporain, celle du rentier notamment décrite par Thomas Piketty dans Le Capital au XXI siècle : il s'agit d'une frange de la population à l'abri de certaines conditions concrètes d'existence comme le travail, et dont la survie économique est indépendante et autosuffisante, plaçant l'hériter dans une position sociale dont les possibilités d'actions sont différentes de celles des autres classes. «Une fois une fortune lancée, la dynamique patrimoniale suit sa logique propre [...]. Avec un patrimoine d'un tel niveau, le train de vie du détenteur absorbe au maximum quelques dixièmes de pourcents du capital chaque année » (Piketty 2013: 442) ${ }^{7}$. Sans aborder la démonstration économique de Piketty, je souligne le langage qu'il emploie pour décrire ses effets, puisque c'est aussi le monde de cette « logique propre » qu'ouvre la richesse héritée du narrateur dans Le fol marbre, une "logique propre » qui autorise tous les crimes. En effet, cette richesse a permis au père d'acquérir, en profitant de la délocalisation et des aléas du marché mondial, et de transformer en loft cossu l'ancienne usine qui servira de théâtre aux actes les plus immondes du protagoniste: "Avant que mon père ne rénove le bâtiment ordinaire du 118, rue de Turenne, il abritait la plus vieille fabrique de chaussures de la ville. Le goût croissant pour les chaussures aux semelles en caoutchouc comme les Vans ou les Nike avait depuis longtemps précipité son personnel à l'état d'équipe squelettique » $(F M, 85)$. Le passé d'usine de la maison du narrateur souligne l'effet mortifère de la mondialisation et de la délocalisation, le texte spécifiant bien qu'il a fallu "rénover» le "bâtiment ordinaire » avant d'y habiter. La rénovation dans le récit tient lieu de signifiant du pouvoir qu'accorde la prospérité du protagoniste, puisqu'elle constitue une manière de transfigurer les lieux et de les adapter à ses douteuses préférences. Aussi, lorsqu'il visite le Château Étage, le narrateur s'empresse-t-il d'« estimer un budget convenable pour restaurer l'endroit menaçant qui avait précédé la cave » ( $F M, 22)$, ce sous-sol qui " avait été un pénitencier avant que le bâtiment ne gagne sa forme définitive » (FM, 21). La «complicité » des conditions économiques avec les crimes du narrateur est ici claire: ses meurtres et ses entreprises culinaires cannibales nécessitant des espaces conçus pour de tels desseins, comme "des éviers aussi larges que des baignoires", permettant de «servir une autopsie en guise de repas puis [de] mettre les assiettes au lave-vaisselle » $(F M, 21)$.

11 Le livre que nous lisons serait donc écrit dans ce langage étrange qu'est le fol marbre. Non pas dans «le fol marbre pur » $(F M, 189)$ du père, cependant, mais dans la version ratée et "plus brouillonne » $(F M, 67)$ du fils, « assemblée à la hâte » $(F M, 129)$. Malgré cette « adaptation "punk" » $(F M, 102)$ de l'originale, la position de force dans laquelle il place son locuteur demeure toutefois évidente dans les passages qui en décrivent l'influence. Le fol marbre se présente dans le texte comme une monstruosité survenue sur les plans du langage et de la construction romanesque. Il contribue, en étant cousu à d'autres morceaux de chair discursive, à donner sa substance monstrueuse à la créature qu'est le narrateur, et à l'asseoir du côté des dominants. Ajoutons à cette domination narrative et économique une dimension raciale, présente dans sa genèse même. Après l'assassinat collectif de son jeune frère Alfonse, vers la fin du récit, le narrateur rapporte une discussion avec son père qui affirme que le délire meurtrier 
s'étant emparé d'Azmir est dû à « une gaffe minuscule » dans son fol marbre $(F M, 186)$. Il explique que

quand il avait conçu la syntaxe d'une confusion appliquée [industriously garbled] qu'il appellerait le fol marbre, il savait qu'elle portait des marques de naissance qui pourraient confiner ses merveilles à un public restreint [cultish]. Comme la plupart des choses venues de France [...] son empire se limitait aux frontières du pays, aux anciennes colonies et à quelques expatriés. Il avait pris trop à la légère le fait que le fol marbre s'effilocherait [would fray] si les auditeurs n'étaient pas nés et n'avaient pas grandi à Paris [born and bred Parisians], s'érodant à mesure que leur dialecte gagnait en épaisseur. [...] Dans le cas d'Azmir, même si le français gauchi et sali qu'il avait parlé pendant son enfance à Alger n'apparaissait pas aux oreilles négligentes plus altéré que la boue [sledge] qui passe pour une conversation à Marseille, il était, en réalité, aussi éloigné du français que le québécois. (FM, 186-187)

12 Ce passage révèle qu'une forme d'« impérialisme linguistique " (Nishiyama 2001 : en ligne) préside au fol marbre; on décrit son «empire» ainsi que la confusion " industrielle» du processus - «industriously» selon le terme anglais qui souligne mieux la dimension économique. Dans ce passage, la narration caricature le discours colonial sur la langue en qualifiant toutes les variations du français hors-capitale de « dialectes ». Le français non-parisien est ainsi associé à l'impureté et à l'épaisseur de la « boue » par opposition à la « clarté » dont Rivarol connaissait le fin mot. Le texte table donc sur un vieux mythe, celui de la clarté française (Swiggers 1987:5-21; voir aussi Mornet 1929) - en anglais, il faut le faire - en dévaluant le « français gauchi et sali » de l'Algérien. Le texte de Cooper, par contraste, fait du narrateur un monstre de la norme blanche et européenne, du français standard en tant que langue dominante. Qu'Azmir soit originaire d'une ancienne colonie française participe évidemment de la signification, le fol marbre se voyant par le fait même associé à l'une des entreprises politiques françaises récentes dont les conséquences furent les plus funestes. La charge coloniale du fol marbre est d'ailleurs réitérée quelques pages plus loin lorsqu'il est décrit comme un "succulent bouillon où l'on pouvait troubler et asservir davantage de langages éclatés » (FM, 189, je souligne).

13 La dimension monstrueuse du narrateur du Fol marbre se construit ainsi en partie par un rapprochement du personnage avec certaines formes sociales de domination (capitalistes, colonialistes) ainsi que par l'exagération de la dimension tyrannique de sa narration sur le récit. Si le monstre chez Cooper est un humain plutôt qu'une créature tentaculaire, c'est parce que l'apparente conformité de ce beau déviant, de ce cannibale raffiné s'élabore dans un mouvement symétrique à celui précédemment nommé, le monstre étant ici, tant sur le plan social que physique, pensé comme une extension de la norme, comme son extrême plutôt que son envers. Le philosophe Alain Denault, dans La médiocratie, analyse les permissions exorbitantes que la large opulence rend accessibles, et résume ainsi leurs implications psychologiques: "être riche, psychiquement, c'est se donner les moyens de manifester aussi aisément et fréquemment que possible ses volontés psychiques : surtout ne pas devoir les contenir dans les coûteux processus du refoulement " (Denault 2015 : 103). Que le riche soit cannibale, pédophile, meurtrier et caetera, chez Cooper se donne à lire comme une interprétation littérale et une hyperbole du phénomène socio-culturel analysé par Denault; la réalisation sans limite des désirs conduit dans $L e$ fol marbre à un éclat de 
violence gore sans frein. Le roman propose ainsi une configuration monstrueuse originale, qui s'apparente à la figure du riche criminel ou de la riche criminelle présente dans l'imaginaire social ${ }^{8}$ contemporain, celui ou celle dont les crimes extravagants sont l'objet d'une complaisance judiciaire et se résolvent par des répercussions minimes sur le mode de vie et les droits du/de la coupable, lorsqu'ils ne demeurent pas carrément impunis. L'œuvre de Cooper fait écho sur cet aspect à la logique immorale des classes dominantes qu'explorent, parmi d'autres, les œuvres d'Elfriede Jelinek, de Bret Easton Ellis, ou encore celle d'une élite fasciste, décadente et hors-la-loi dépeinte par Pasolini dans Salò, ou les 120 Journées de Sodome ${ }^{9}$.

\section{La dévoration des catégories}

La fonction critique du monstre serait un peu mièvre si elle se résumait à présenter celui-ci comme une simple version exagérée des normes socioculturelles contemporaines. Bien qu'elle permette de saisir plusieurs éléments de la construction du monstrueux à l'œuvre dans le roman de Cooper, cette interprétation se révèle impuissante lorsqu'on cherche à comprendre les comportements les plus abjects du narrateur, comme ses penchants cannibales, homicides, nécrophiles, incestueux ou pédophiles. On a longtemps pensé le monstre comme l'Autre absolu du sujet, comme son envers, ce qui s'oppose à son identité. Or l'œuvre de Dennis Cooper, c'est ce que je voudrais désormais montrer, nous invite à lire un monstre qui échappe à la conception unitaire du sujet classique, de l'être humain et du genre sexuel. Le Fol marbre met en scène la fin de l'individualité du monstre en faisant naître dans le récit un genre «monstrueux », en ce qu'il fuit et défait les catégories sociales de l'identité sexuelle. Le monstrueux comme essence ne suffit plus, et pour comprendre le roman, il faut s'ouvrir au monstre comme processus, au monstre qui dissout la notion usuelle d'identité et les oppositions de la pensée dualiste. Cette voie de réflexion, ouverte par la critique queer, me permettra de lire des compositions sexuelles et corporelles monstrueuses dans le roman qui ne se résolvent pas dans une cohérence identitaire ou psychologique. Comme le dit le narrateur lui-même en s'adressant au lecteur, "pour citer et nuancer Rimbaud, je te mets au défi de me prouver que je ne contiens pas des multitudes » $(F M, 175)$.

15 Le premier indice de cette dispersion du monstrueux est la dimension sérielle qui marque les personnages du roman, qui pourraient en un sens tous être considérés comme des monstres, voire des morceaux d'un même monstre narratif. En effet, le mal chez Cooper est toujours partagé, sans pour autant être dilué. En analysant seulement le protagoniste, il est toutefois possible de montrer que Le fol marbre donne à lire un genre monstrueux, insaisissable dans la description négative qui en est faite, et tout aussi difficile à assigner dans son rapport détaché au désir que dans son attirance pour les actes les plus terribles. Le roman met en scène une série de personnages que le narrateur désigne comme " chacun une nuance de gay » $(F M, 58)$. Malgré ce que laisse entendre cette affirmation, qui pourrait faire croire que nous avons affaire à un roman qui thématise l'homosexualité, l'écriture de Cooper entretient un rapport conflictuel avec les identités collectives, rapport qui s'est longtemps traduit par une méfiance envers ses textes dans les études sur l'homosexualité en littérature (Lev 2006 : 20-22). Rien n'est simple chez Cooper, comme le montre la suite de la phrase, qui spécifie: « chacun est une nuance de gay - en tout cas c'est ainsi que je les nommerai par paresse pour 
l'instant" (FM, 58, je souligne). Tout au long du roman, le narrateur multiplie les remarques du genre : « si j'étais à moitié aussi gay que vous l'imaginez » (33), « si j'étais gay ou, puisque vous insistez, si j'étais complètement gay, j'aurais... enfin à vous de me le dire. Je ne suis pas assez gay pour le savoir » (64), « puisque par prévention je m'étais étiqueté comme gay, j'étais encore soumis à la ligne du parti homosexuel " (97), plus loin il parle de «mon besoin de piétiner votre ${ }^{10}$ empressement à me déclarer gay avec une pointe de psychose» (121). Notons que, dans cette dernière citation, il n'est pas facile de savoir à qui va la psychose : au narrateur monstrueux ou au lecteur implicite qui cherche absolument à diagnostiquer sa sexualité et sa perversité? À l'homosexualité comme identité, le narrateur oppose son incomplétude («si j'étais complètement gay", "si j'étais à moitié aussi gay»), parodiant ainsi les discours, notamment scientifiques, qui entendent faire de la sexualité une donnée observable et quantifiable - pensons à l'échelle de Kinsey, conçue pour marquer «scientifiquement » l'orientation sexuelle d'une personne sur une échelle numérique, ou aux études récentes qui tentent de trouver un "gène gay» dans l'ADN humain. Comme le montrent les passages cités, le texte utilise la catégorie " gay " pour constamment s'en distancier, souligner l'inadéquation du personnage à ce terme qui, pour citer Judith Butler, «n'arrive pas à l'exhaustivité, [...] parce que le genre n'est pas toujours constitué de façon cohérente ni conséquente selon les différents contextes " (Butler 2005 : 62-63), ce que le reste du texte s'attache à illustrer.

En neutralisant la grille de lecture (strictement) homosexuelle et en joignant au désir du narrateur pour les garçons (mineurs) une envie de les tuer ( «je venais presque de décider que pour me convenir Serge devrait mourir» [FM, 19]) et de les dévorer («à un moment donné, l'un de nous, probablement moi, voulut aller chercher un bloc de couteaux de cuisine, pensant que nous pourrions taillader Alfonse et nous débarrasser de lui puis retirer ses entrailles et les avaler comme des singes " [ $F M, 150])$, le roman de Cooper associe l'homosexualité à un certain nombre de perversions (ajoutons-y la pédophilie et l'inceste) que les discours homophobes ont employées pour la rendre monstrueuse. Le processus de démonisation de l'homosexualité qu'entame le roman vise cependant un tout autre objectif politique que le discours homophobe. En mettant en scène des comportements sexuels socialement considérés comme inacceptables, le texte montre que l'homosexualité, comme tout autre discours identitaire ou de genre, se construit sur l'édification d'une frontière, par le rejet négatif de ce qu'elle n'est pas (perverse, cannibale, pédophile, etc.). En ramenant en avant plan ce qu'elle exclut pour établir ses fondations, Cooper révèle son statut de simple catégorie ainsi que le processus de cette fabrication. C'est ainsi moins en questionnant directement les catégories traditionnelles « homme » et «femme » que le roman s'intéresse à critiquer le genre comme source d'identification, qu'en rejetant une identité elle-même minoritaire, mais dont le fonctionnement par réduction et par simplification de la vie des êtres selon leurs corps, leurs désirs et leurs actions correspond à la critique butlérienne du genre. Dans une analyse fondamentale de la poésie de Cooper, Pedro Antonio Férez Mora propose une interprétation semblable en avançant que la sexualité limite dans son œuvre fait office de :

mechanism to denounce the fossilisation of sexual practices to just those permitted in "the charmed circle of sexualities", to borrow Gayle Rubin's words: heterosexual, married, monogamous, procreative, non-commercial, in pairs, in a relationship, same generation, in private, no pornography, bodies only, vanilla. [...] From this viewpoint, Cooper's portrayal of non-normative sexualities [...] aim to block the traditional, to interrupt the normalisation of only certain forms of sexualities - 
those sanctioned by the tradition - to the detriment of others (Férez Mora 2013 :

93).

17 L'objectif d'une telle stratégie littéraire n'est pas de rendre les sexualités voraphile ${ }^{11}$ ou pédophile acceptables, mais d'utiliser ces signifiants socialement abjects et monstrueux pour révéler les mécanismes d'édification de toute norme sexuelle. Le fonctionnement de l'abjection dans le texte cooperien prend ainsi une dimension politique, déjà théorisée dans l'ouvrage canonique de Julia Kristeva, chez qui l'abject œuvre comme "ce qui perturbe une identité, un système, un ordre " en incarnant "l'entre-deux, l'ambigu, le mixte » (Kristeva $1980: 12)$.

18 La faille dans la normalisation des catégories sexuelles qu'ouvre le texte de Cooper affecte aussi le désir des personnages, et s'étend plus largement à l'expression de la sexualité dans le roman. Le sexe, pour le narrateur du Fol marbre, n'offre aucune vérité, aucune transcendance particulière, «sa gloire et son éclat sont le mensonge le plus merdique du monde ", pour le dire avec Serge, « pire que le canular du père Noël » (FM, 75). Plutôt que d'être décrit comme une expérience de communion entre deux individus, voire comme une manière de soulager ses envies sexuelles, le sexe n'y est rien de plus qu'une série de processus techniques disparates, une enfilade de scripts sexuels sans horizon de référence composant une "chorégraphie porno » $(F M, 143)$ inscrite dans aucune cohérence d'ensemble ${ }^{12}$, allant de la masturbation à la torture souvent consentie - du corps vif ou bientôt mort, et qui vise à produire une jouissance selon la logique fragmentaire proposée par Jean-Ernest Joos. Le critique, dans son analyse du roman Frisk (1991), rappelle en effet que chez Dennis Cooper « le fragment d'expérience rencontre sa limite dans l'impossibilité de faire [...] synthèse » (Joos 1999 : 96). La jouissance n'est ainsi jamais présentée comme la culmination du rite sexuel. Elle est sans synthèse, sans hapax, autant dire qu'elle survient trop tôt, trop tard, ou pas du tout: " il manquait dans le meurtre un point fort ou un arc dramatique qui m'aurait permis de penser : “c'est maintenant ou jamais" et de me masturber jusqu'à ce que la scène ne soit plus qu'un contrecoup » $(F M, 163)$. Le discours du sexe serait suivant cette logique une vaste construction frauduleuse, selon le narrateur qui «trouve les termes en rapport avec le sexe mal accordés à [s]on décorum» $(F M, 121)$. Un passage en particulier, dans lequel il exprime son désir pour Serge, montre bien comment le roman met en texte la méfiance du personnage envers la sexualité. On y lit :

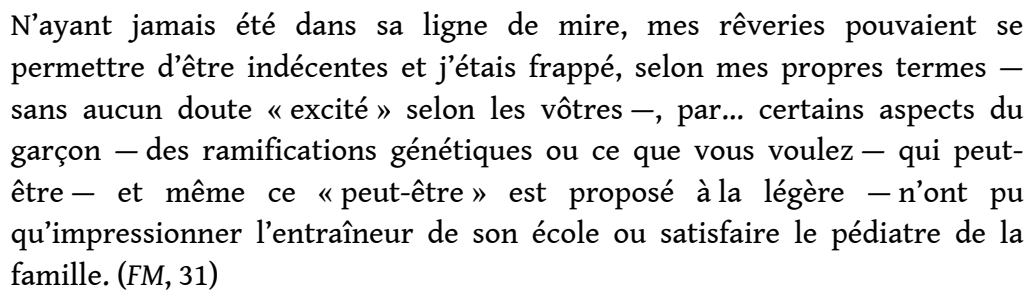

19 La figure de l'épanorthose, cette correction qui survient lorsque le narrateur traduit son expérience dans le vocabulaire conventionnel du sexe («excité » plutôt que «frappé »), jointe à l'hésitation qui marque le paragraphe ainsi qu'aux nombreuses spécifications entre tirets, témoignent bien de sa réticence à mettre des mots sur un désir qui entend rester insaisissable et indéfini, et inversement, de l'incompétence du langage à nommer l'ordre du désir. Que ce soit des "ramifications génétiques " qui provoquent son attirance pour Serge pousse à sa limite la logique du désir du narrateur, puisque c'est l'invisible, l'infiniment petit du gène qui est présenté comme 
attirant pour lui, plutôt que le sexe du garçon, par exemple. Encore une fois, le texte déjoue la logique normative de l'homosexualité et la différenciation homme/femme sur laquelle elle repose (Férez Mora 2013), en plus de reproduire une logique propre à la «technoscience contemporaine » analysée par Paul B. Preciado dans Testo-junkie: sexe, drogue et biopolitique. L'auteur analyse longuement dans cet ouvrage l'effet épistémologique de l'apparition de «la psychologie, la sexologie et l'endocrinologie [qui] ont établi leur autorité matérielle [en] transformant les concepts de psychisme, libido, conscience, féminité et masculinité, hétérosexualité et homosexualité, en réalités tangibles, substances chimiques, molécules commercialisables, corps, biotypes humains, valeurs marchandes gérables par les multinationales ", et dont la "grande réussite [...] est de transformer notre dépression en Prozac, notre masculinité en testostérone, notre érection en viagra, notre fertilité/stérilité en pilule, notre sida en trithérapie » dans une "production en autofeed-back» (Preciado 2008 : 33-34). Dans le passage commenté précédemment, le désir du narrateur parodie cette transformation, qui place un élément biologique ou pharmacologique comme source de différentes réalités culturelles, en faisant de la "ramification génétique » l'origine de son attirance sexuelle ${ }^{13}$. Cette image, en plus de servir à contourner les catégories binaires liées au genre (masculin ou féminin), instaure un autre motif, primordial dans l'œuvre de Cooper comme l'attestent les travaux de Pedro Antonio Férez Mora, celui de la décorporalisation (Férez Mora 2014 : 78). En se décrivant, par cette métaphore génique, comme attiré par l'infinitésimal, par le degré zéro du corps humain, le narrateur avance de manière ici caricaturale que ce corps, pensé comme ensemble cohérent d'organes et porteur d'une identité, est une convention à laquelle on peut bien refuser d'adhérer.

20 Le corps est souvent décrit comme le premier (et le dernier) repaire de l'identité. Dans le Fol marbre toutefois ses limites sont perméables et les distinctions entre son extérieur et son intérieur, entre sa vie et sa mort et, par extension, entre l'humain et l'inhumain sont des pièces qui supportent mal l'opposition. Le corps chez Cooper n'est pas monstrueux à la manière de celui du monstre de Frankenstein ou de celui du comte Dracula, qui causaient par leurs points de sutures et leurs longues oreilles l'émoi de la classe bourgeoise au XIX ${ }^{e}$ siècle ; il l'est parce qu'il s'écrit à l'extrême de l'humain, dans cette zone limitrophe où le corps est poussé par le texte et où il risque toujours de se dissoudre dans la somme de ses parties. Cette réflexion sur le corps, nous le verrons, permet de mieux comprendre le rôle de la scène gore dans l'esthétique cooperienne, puisque c'est dans la violence extrême que les limites du corps s'y expérimentent. Comme le synthétise bien Joos, nous verrons que le texte présente, par le gore, une :

réduction du corps à une chose sur laquelle va s'accomplir le projet d'une abolition de toute limite, notamment entre la vie et la mort, entre l'image et la réalité, le sexuel et le non-sexuel. Ainsi, certains personnages «objectifient" leur corps au point de se regarder en train d'être manipulés par d'autres, au point de désirer leur propre mort dans un rapport sexuel. D'autres n'éprouvent de désir que s'ils peuvent fantasmer la mort de leur partenaire sexuel, ou sont amenés à érotiser la mort et, par-delà la mort, l'intérieur du « corps assassiné » (Joos 1999 : 94).

21 Alfonse, le frère du narrateur, est un jeune garçon qui distingue mal la réalité de la fiction et qui métamorphose sa physionomie grâce à Photoshop. Il est décrit comme " une jeune sirène coincée entre les pages imprimées de ses héros illustrés, où il rêvait de voler avec des chaussures à réacteurs, de changer de genre en appuyant sur un bouton, et notre dimension plus pesante où il survivrait mais sans trouver sa place » 
$(F M, 87)$. Cette « dimension ", qualifiée de manière prémonitoire de " pesante », il rêve d'ailleurs de la porter sur lui, jusqu'à en être anéanti ; s'il avait à choisir sa mort, il aimerait que ce soit "écrasé par un rouleau compresseur", car il "fréquent[e] plusieurs salons de discussions en ligne remplis de gamins tout aussi renfermés et de prédateurs mensongers qui adulaient ce qui n'a pas plus d'épaisseur que le papier " (FM, 88). Il avoue lui-même : "de plus, quand je me sens irréel, le... sexe est meilleur pour tous ceux qui sont impliqués » $(F M, 141)$. Le meurtre d'une extrême violence dont Alfonse est victime est probablement le morceau de littérature le plus éprouvant que j'aie eu à lire. Narré par les yeux du narrateur, qui assiste en retrait au dérapage d'Azmir et de François baisant brutalement et jusqu'à la mort la carcasse de son frère, ce passage est peut-être le seul moment dans tout le roman où le protagoniste perd le regard « de marbre » qu'il pose habituellement sur les événements du récit. Dans cette partie du texte, il fait des détours, s'arrête momentanément de narrer la scène pour se souvenir de la mort de son père, sa mordante ironie s'émousse, on le sent ébranlé, impuissant. Quelque chose de mélancolique qui s'instaure dans le ton de ce psychopathe rend la scène véritablement perturbante pour le lecteur ou la lectrice. L'horreur de la scène atteint littéralement le texte, puisque plus les atrocités s'accumulent, plus sa narration se défait tranquillement : «Pendant un moment je vais à peine parler. Je commence peut-être à ressembler à un enfant qui reproduit avec sa bouche les rugissements et les accélérations du moteur de ses jouets » (FM, 147). Il ajoute plus loin: "je manque de spontanéité pour vous rapporter ce qui arriva à Alfonse, il faudra faire avec l'enchaînement d'actions que je vais raconter » $(F M, 152)$.

Comme pour rendre la scène plus supportable pour lui ou pour nous, les descriptions $\mathrm{du}$ sort physique d'Alfonse opèrent une décorporalisation de ce dernier, qui apparaît dans le texte sous forme de comparaisons. Le narrateur "tripote le cul d'Alfonse comme si sa graisse et son muscle constituaient les éléments pivotants d'un Rubik's Cube » (143), le garçon "ressemblait à une radiographie » (149), "ses articulations [étaient] semblables à celles d'un mannequin » (149). Cette objectification transforme le corps d'Alfonse en un amas de choses inanimées, en un ensemble de dispositifs sans synthèse qui déshumanisent le personnage, le décomposent avant sa mort en le transformant en une « infrastructure humaine » (209), syntagme qui met l'accent sur la dimension technique, construite et contingente de la notion d'« humain» et de son rapport au corps, révélant ainsi que la première ne saurait se réduire au dernier, dans une adéquation pure, simple et limitée entre l'anatomie et l'identité. À un autre endroit, pendant le récit du meurtre, on lira que « son souffle était un courant d'air, ses gémissements et plaintes les grincements de son visage, et son crâne un treillis sur lequel de la chair avait poussé et pris forme humaine » (FM, 151). D'une manière semblable, la dernière métaphore place l'humain, le corps comme le résultat d'un procédé de construction qui se fonde sur une série d'objets inanimés, dont le treillis sur lequel, à la manière du végétal, pousse la forme humaine d'Alfonse. Dans Le fol marbre, c'est le treillis qui anticipe la chair, puis l'humain ; « les romans de Dennis Cooper sont “posthumains ${ }^{14 "}$ ", puisque l'évidence d'une nature humaine y fait défaut» (Joos 1999 : 97). Le texte gore nous révèle crûment ces mécanismes en décomposant le corps d'Alfonse sous nos yeux.

23 Un élément précis de l'imaginaire social se voit questionné dans les violences du Fol marbre: celui d'une "vérité » supposée du genre qui se situerait dans l'anatomie, selon la logique de la « matrice hétérosexuelle » définie par Judith Butler comme « un modèle 
discursif/épistémique hégémonique d'intelligibilité du genre; dans ce modèle, l'existence d'un sexe stable est présumée nécessaire à ce que les corps fassent corps et aient un sens, un sexe stable traduisible en un genre stable» (Butler 2004: 66). La description des infamies faites au corps d'Alfonse peut ainsi se comprendre comme une tentative de pousser à bout la logique folle du discours hétéronormatif, en cherchant dans le corps cette vérité de l'identité sexuelle dont la « matrice hétérosexuelle » pose l'existence. Sans surprise, on ne la trouvera pas chez Cooper, le texte exposant plutôt les conséquences funestes et brutales de la recherche elle-même. Le meurtre extrême devient donc un prétexte pour questionner de manière radicale la matérialité du corps, dans une tentative de résoudre «la querelle idiote d'Alfonse avec la physique » (FM, 190) en explorant les zones où l'humain se défait, où la distinction entre la vie et la mort, le masculin ou le féminin ne sont plus évidentes. Ou pour le dire avec Halberstam, la partie la plus gore du texte nous rappelle que :

gender is monstrous in the horror [...] and it exceeds even the category of human. The genders that emerge triumphant at the gory conclusion of a splatter film are literally posthuman, they punish the limits of the human body and they mark identities as always stitched, sutured, bloody at the seams, and completely beyond the limits and the reaches of an impotent humanism $(S S, 141)$.

24 Et si le corps se défait d'une manière dégoûtante, si le narrateur le dépeint "dépérissant, se liquéfiant et clapotant contre le squelette de mon frère avant de devenir une gelée et d'emballer sous plastique ce qui était devenu un cratère » (FM, 153), c'est pour donner par la fiction une forme à la violence d'une idéologie qui repose sur « le produit d'une division sexuelle de la chair selon laquelle chaque organe est défini par sa fonction» (Preciado 2008: 44) et qui permet de situer le siège de la différence sexuelle à un endroit plutôt qu'à un autre. C'est dans cet entre-deux informe, dans la position abjecte qui abolit la division entre intérieur et extérieur du corps, que le narrateur trouvera une forme de beauté : « ce qui avait composé le visage d'Alfonse à partir de son squelette, sa peau et son cartilage arrêta de fonctionner, mais je trouvais qu'il avait l'air incroyable » (FM, 154).

Dès qu'Alfonse se déshabille devant le narrateur, on peut lire que « son corps aurait pu être un bocal à forme humaine et aux teintes chair les plus pâles" $(F M, 142)$, comparaison qui annonce que la scène gore cherchera à comprendre ce que contient ce corps-bocal, qu'on décrit si souvent comme une enveloppe. Ce que Jack Halberstam décrit dans son analyse du splatter film comme une " preoccupation with [...] not simply the external monstrosities of the body but the increasingly voyeuristic quest to show what lies below the skin» (SS, 139) pousse, comme je l'ai dit, à bout la logique hétéronormative délirante du corps qui propose de déterminer un genre (intérieur et invisible) à partir d'un sexe (extérieur et visible). Que les organes sexuels d'Alfonse soient mutilés jusqu'à en être complètement déformés n'est pas anodin : l'assassinat gore est une manière, chez Cooper, de tout aplanir dans la dimension du visible, en plus de mettre à l'épreuve la découpe "intérieur/extérieur " des corps, fondamentale comme on le sait dans l'établissement de la différence sexuelle (Preciado 2000 ; Férez Mora 2014). Ainsi, les descriptions de sang qui gicle, d'organes qui éclaboussent en "défonç[ant] de nouveaux raccourcis là où ils en avaient besoin» ( $F M, 157)$ se multiplient, dans un surgissement furieux du dedans sur le dehors, et ce, jusqu'à ce qu'il devienne impossible de distinguer ce qui appartient à l'un ou à l'autre. Le corps, alors, « para[ît] changer de métabolisme » $(F M, 153)$, pour se fondre en " une forme non définie » $(F M, 154)$. Le cadavre d'Alfonse finira d'ailleurs aplati, fusion littérale s'il en 
est une de l'intérieur et de l'extérieur dans une même surface, qui ne nous laisse plus que devant « the irreductible materiality of the body " (Lev $2006: 29)$.

Que reste-t-il à dire de cette narration qui donne à voir le monstrueux, qui endosse l'abject et repousse les frontières du dicible? Quel savoir peut se dégager de la violence gore faite au corps? La pulsion scopique et discursive qui anime cette partie du texte, qui dit et montre tout, n'est-elle pas, finalement, une tentative de penser la mort, un questionnement sur son origine et sur la possibilité du langage à la saisir ? Il n'est pas aisé, dans Le fol marbre, de savoir quand la mort survient : « il m'était difficile de savoir quand [la mort] a eu lieu exactement, la violence était tellement frontale et les battements de son cœur une telle aiguille dans une botte de foin» (FM, 163). Au moment le plus violent de son crime, le narrateur dira d'ailleurs : «je n'arrivais pas à penser, et même cette insouciance fut annulée quand toutes les célébrités mortes dont j'avais cherché sur Google les photos prises à la morgue s'amoncelèrent depuis le fin fond de ma mémoire et claquèrent des doigts devant mon visage " (FM, 157). Pour concevoir la mort de son frère devenu méconnaissable, le narrateur ne peut que se rabattre sur les images médiatiques de célébrités défuntes, qui seules peuvent prétendre offrir une image fixe et stable de la mort. Pour reprendre les mots de Judith Butler dans Ces corps qui comptent, Le fol marbre nous montre, en décrivant la désagrégation innommable à laquelle est soumis le corps d'un jeune garçon, mais aussi en marquant la résistance du narrateur à se définir comme "gay" et plus généralement sa méfiance envers le discours conventionnel sur le sexe et le désir, que :

la construction du genre se réalise au moyen d'exclusions, de telle sorte que l'humain n'est pas seulement produit en opposition à l'inhumain, mais à travers un ensemble d'exclusions, d'effacements radicaux, auxquels est refusée, à strictement parler, la possibilité d'une expression [rearticulation] culturelle. Par conséquent, il ne s'agira pas d'affirmer que les sujets humains sont construits, car la construction de l'humain est une opération différenciatrice qui produit du plus et du moins «humain», de l'inhumain et de l'humainement impensable. Ces sites d'exclusion viennent borner l' «humain» comme son dehors constitutif, et hanter ses frontières comme la possibilité persistante de leur perturbation et de leur réarticulation. (Butler 2009: 21)

En faisant apparaître dans la représentation romanesque les "exclusions» sur lesquelles le discours homosexuel se construit comme une nouvelle norme, l'esthétique cooperienne révèle le travail de stratification et de différenciation à l'origine de toute catégorie. Sa démarche littéraire vise ainsi à faire surgir dans l'imaginaire ce qu'il rejette - consciemment ou non - dans sa perception de la sexualité (Férez Mora 2013 : 83-84). Le genre monstrueux, dans Le fol marbre, incarne plus que l'antithèse de la norme, puisqu'il remet en question le processus de formation de toute norme, ou pour le dire avec les mots de Sam Bourcier, les livres de Cooper (tout comme le cinéma de Bruce LaBruce dont il est question dans ce passage de Queer zone) "proposent une critique des effets normatifs et excluants d'une identité gaie dominante, blanche, issue des classes moyennes et volontiers assimilationniste " (Bourcier 2011 : 33). Le statut du genre dans le roman, qui se méfie des définitions et se construit comme monstrueux en dépeçant et ravageant le corps d'Alfonse, autrement dit nous rappelle que " le sujet est constitué à travers la force d'une exclusion et d'une abjection, qui produit un dehors constitutif [...], un dehors défini comme abject qui est, finalement, "à l'intérieur" du sujet, comme la répudiation qui le fonde " (Butler 2009: 17). Là où Butler s'arrête, Cooper prend la plume pour explorer, dans l'espace de la littérature, les répudiations qui fondent la normalité sur le cadavre de l'abject ou des petits garçons, en proposant 
par la mise en texte de la monstruosité, une exploration des limites des catégories de genre, du corps et de l'humain, sur lesquelles la culture hétéronormative fonde ses assises.

\section{Le monstre créé}

28 Le troisième et dernier changement dans l'imaginaire du monstre que traduit Le fol marbre touche la dimension productive de la monstruosité. Le monstre du roman est d'abord présenté comme le fruit d'une création, comme un être dont les racines auraient été engraissées (volontairement) par des humeurs morbides, sa monstruosité se présentant comme l'effet d'un encadrement psychologique et architectural, sur lequel je reviendrai. Les limites du monstrueux, sa séparation franche d'avec la norme étant contestée tout au long du roman, comme je l'ai déjà montré, j'avancerai maintenant que la possibilité de générer du monstrueux implique une performativité plus large du geste littéraire, souvent présente - de manière fantasmatique - dans l'œuvre de Cooper, qui vise à impliquer le lecteur ou la lectrice imaginaires dans ses enchevêtrements monstrueux, à les rendre « complices » de ses fantasmes horrifiques.

29 Les recherches de Jean-François Chassay soulignent, en régime contemporain, un changement de paradigme dans l'imaginaire du monstrueux, changement dont la figure du clone ou du monstre scientifique est symptomatique : « nous passons de plus en plus du monstre "né" au monstre "créé" » (Chassay 2014: 8). Le monstre du Fol marbre semble avoir intégré cette logique, puisqu’à plusieurs moments dans le livre, le narrateur met l'accent sur les événements qui ont contribué à le "faire " monstre, et suite auxquels ses envies de chair humaine se sont affirmées. Il raconte, par exemple, que pendant son adolescence :

un pro de l'informatique [...] remplit les marque-pages de mon moteur de
recherche de nouveaux liens vers les sites de meurtriers de toutes sortes
spécialisés dans les enfants, supposant, avec une certaine prescience, que je
leur ferais un trop bon accueil pour me demander comment ils étaient
apparus. Quand mes clics me ramenèrent systématiquement vers un
cannibale allemand, on mit en place d'autres pièges du même acabit. Par
exemple, un récent chat auquel j'avais pris part sur le plus grand site de fans
de Klaus Freeh où je partageais mon rêve de transformer Alfonse en une
bouchée n'était pas la mêlée générale que j'espérais, mais une inquisition
menée par mon père. $(F M, 119)$

30 Ainsi le père du narrateur aurait-il placé dans l'ordinateur du narrateur des sites web faisant office de "pièges » qui, joints à l'action du fol marbre, paraissent destinés à pervertir son fils, à faire de lui un monstre. La figure fictive du cannibale Klaus Freeh, un « scientifique en physique des particules qui pensait que s'il parvenait à humaniser sa compréhension des nanoparticules, il deviendrait le premier génie cannibale du monde » ( $F M, 96)$, tout comme celle de Pierre Clémenti dans le film I cannibali de Liliana Cavani ( $F M, 238-240)$, font office dans le texte de modèles générant l'identification du narrateur adolescent tout en éveillant chez lui ses désirs anthropophages. C'est « grâce à ce contrôle à distance [que] ma relation avec Alphonse fut revue et corrigée en l'espace de plusieurs mois » (FM, 118, je souligne), affirme-t-il dans une phrase qui détourne l'expression éditoriale commune. Le fait que cette expression, " revu et corrigé », soit ici accordée avec le verbe "être" signale d'ailleurs la transformation plutôt que la 
forme achevée, dans une perspective visant à insister sur le rôle des différents dispositifs et des récits cannibales dans le devenir-monstrueux du narrateur. La fiction a en effet souvent un pouvoir très fort de transformation de l'être humain ou de ses comportements chez Cooper, qui déploie fréquemment un imaginaire de l'action ou de l'effet direct du livre sur le lecteur ou la lectrice. Un autre récit enchâssé dans la diégèse témoigne de la force d'action que possède la littérature sur le réel au sein de l'univers romanesque cooperien, celui d'Arval Benicoeur, qui aurait retrouvé le dernier roman perdu du Marquis de Sade, L'égarement de l'infortune, et qui après l'avoir lu se serait emmuré avec quinze squelettes d'enfants avant de se pendre, réalisant ainsi les "scénarios de la fiction extrémiste de Sade » (FM, 179).

31 Ce qui m'intéresse dans cette fabrique du monstre par un Dr Frankenstein dématérialisé et disséminé, dans ce processus de transformation monstrueuse subi par le narrateur depuis l'enfance en raison d'une exposition à des œuvres (web, cinématographiques) qui le pervertirent, n'est pas tant l'influence véritable que peuvent avoir ces médias sur les enfants que le rôle joué par les espaces, le milieu de vie et la culture dans l'institution des désirs monstrueux de ce personnage de roman. J'ai précédemment commenté la fonction des lieux comme espaces permettant l'expression monstrueuse du narrateur, en montrant que son manoir pharaonique se révèle être un endroit où ses désirs les plus ignobles peuvent se réaliser à l'abri des interdits sociaux. Je développerai davantage cette idée en analysant comment la maison, qui s'apparente à une " pornotopie », catégorie spatiale et architecturale théorisée par Paul B. Preciado, participe aussi à la production d'un narrateur (et d'une énonciation) monstrueux. Le concept d'espace pornotopique, élaboré par Preciado dans Pornotopie: Playboy et l'invention de la sexualité mutimédia, à partir des travaux du critique littéraire Steven Marcus et de ceux de Michel Foucault sur l'hétérotopie, est défini ainsi par le philosophe:

Ce qui caractérise une pornotopie est sa capacité d'établir des rapports singuliers entre espace, sexualité, plaisir et technologie (audiovisuelle, biochimique, etc.), en altérant les conventions sexuelles des genres tout en produisant la subjectivité sexuelle comme un dérivé de ces opérations spatiales. [...] L'ensemble [des] pornotopies ouvre une brèche dans la topographie sexuelle de la ville, provoque des altérations des codes normatifs du genre et de la sexualité, des techniques du corps et des pratiques de production du plaisir (Preciado $2011: 119-120$ ).

Le Château Étage ainsi que le loft parisien du narrateur, dont l'un est le double défiguré de l'autre, sont des exemples si parfaits de pornotopies qu'on pourrait se demander si Cooper n'a pas lu l'ouvrage de Preciado. En effet, par « une coïncidence très étrange ", mentionne le narrateur, "la maison où j'avais passé la majeure partie de ma vie dissimulait aussi un maigre domaine secret mal éclairé, également façonné à partir des creux d'une demeure en apparence normale, et, même si ce lieu était bien moins complexe que celui du château, je peux vous assurer qu'il était tout aussi pernicieux » $(F M, 44)$. Ces «tunnels secrets » $(F M, 44)$, à la manière de la pornotopie, sont munis de dispositifs audiovisuels permettant au père du narrateur (et à celui de Serge, dans le Château Étage) d'espionner leur progéniture: les murs y sont "envahis de judas, chaque encoche munie d'une lentille panoramique offr[ant] une vue de chaque recoin du loft correspondant [...] aspirant nuit et jour les images des pièces sans défense des lofts " (FM, 115). Que la prise d'images pornographiques filmées dans la chambre du narrateur et de son frère soit décrite comme une forme de vampirisme («aspirant » les " pièces sans défense ») accentue « les altérations des codes normatifs du corps et de la 
sexualité » caractéristiques de la pornotopie selon Preciado, en rendant acceptable, dans cet espace secondaire, une réalité interdite dans l'espace principal (la pornographie juvénile incestueuse), tout en associant cette curiosité déviante à un comportement monstrueux (vampirique). Par cette technologie de visibilité pornotopique, le roman élabore le rôle du château et du loft dans les compositions sexuelles tordues d'un récit où les pères enregistrent le viol de leurs enfants pour les envoyer par email à d'autres hommes, une composition cybernétique de la sexualité où elle se voit surveillée, enregistrée et reproduite grâce à un ensemble de médiations audiovisuelles et de jeux d'espionnage qui construisent « une fiction théâtralisée de la sexualité15 " (Preciado 2011: 119). Un autre trait définissant l'espace pornotopique, celui d'une production de «la subjectivité sexuelle comme un dérivé [des] opérations spatiales ", se voit de surcroît directement mis en lumière par le narrateur, lorsqu'il décrit ainsi la dimension générative du loft sur sa propre personnalité monstrueuse : «j'ai passé tellement de temps encerclé par les œuvres que mon père achetait [...] qu'il est fort probable que ces goules fixes m'aient lavé le cerveau et transformé en une œuvre humaine rusée [...] qui, comme leurs soi-disant créateurs post-conceptuels, pense que tout sujet lié aux émotions [...] n'est pas assez cool pour être représenté » $(F M, 60)$. Ici, la séparation nette entre la nature du sujet et son milieu - constitué d'œuvres d'art décorant le loft - se voit brouillée par une narration qui opère un télescopage et transforme textuellement le protagoniste en «œuvre humaine ", en un produit de la pornotopie dans laquelle il a grandi et qui lui aurait "lavé le cerveau ». Les frontières de l'humain ne sont, comme nous l'avons déjà vu, jamais stables dans Le fol marbre, et le surjet entre l'intériorité émotionnelle du narrateur et son environnement extérieur, entre le corps et les objets qui l'entourent témoigne de cette reconfiguration du vivant et de l'inanimé, désormais portée par un rapport de coextensivité plutôt que d'étanchéité. Le monstre ne préexiste pas à sa création, il est le fruit d'une production, d'un devenir dans lequel les lieux, les espaces pornotopiques - qui se caractérisent par la permission de comportements que l'espace principal considère comme inacceptables - sont fondamentaux.

33 Notons que les pornotopies du Château et du loft ne se résument pas au décor dans lequel se déroule l'action du roman. Par le biais d'une comparaison constante entre le langage étrange employé par le narrateur, ce «fol marbre », et les couloirs secrets de la maison, le roman fait de son énonciation elle-même une forme pornotopique. Dès le début du roman, dans l'un de ses nombreux commentaires métanarratifs, le narrateur confesse sa confusion: "il se trouve que je suis compliqué, ou plutôt, il se noue un trajet étrangement tortueux entre ce que j'ai l'intention de dire et ce que je devine de mes pensées» $(F M, 65)$. La métaphore employée ici, celle du «trajet étrangement tortueux ", associe le fol marbre (dont il est question pour la première fois à la page suivante) à une construction architecturale qui fait penser à un labyrinthe, dans lequel la parole du narrateur, pour paraphraser le texte, suivrait une trajectoire sinueuse. Plus loin, cette dimension matérielle, concrète que le texte donne à la narration du protagoniste est amplifiée. Dans une adresse au lecteur implicite sur le mode impératif, il dit alors: "essayez d'imaginer que ma voix est plus concrète et physiquement imposante que le livre [...]. Disons que... c'est un château, puisque ce décor est encore frais. Disons que pendant que vous lisiez ou, en fait, avez cru lire, vous vous promeniez dans mon château en étant assez attentifs pour trouver votre chemin dans ce passage secret que je décrivais » $(F M, 81)$. La correspondance entre les couloirs labyrinthiques de château et le fol marbre du narrateur est ici réitérée. En faisant de sa narration, par 
l'utilisation de métaphores, l'équivalent des passages secrets qui se trouvent derrière les murs du manoir, le narrateur place en quelque sorte le lecteur implicite dans cette architecture. Lire, si l'on file la métaphore, revient à se promener dans le labyrinthe que fait naître l'énonciation. Par cette image, le narrateur se donne à la fois le rôle de Dédale ( «je serais l'architecte du bâtiment et mon histoire, sous sa forme actuelle, en constituerait le plan » [FM, 81]), et à la fois celui du minotaure, cette bête qui se tapit au détour d'un des passages de ce méandre. Mais qu'est-ce qu'impliquent, sur le plan littéraire, ces formulations, qui pourraient bien ne se résumer qu'à des métaphores?

34 Si le texte montre minutieusement comment les lieux et les récits produisent, génèrent, fabriquent le monstrueux, et que le lecteur ou la lectrice « est " placé.e dans une narration qui, nous dit-on, est l'équivalent littéraire de ces espaces pornotopiques, n'est-il pas possible qu'il ou elle devienne aussi... un monstre ? Ainsi, peut-être que l'ultime fantasme du narrateur (et dans une certaine mesure du roman) est de faire du lecteur ou de la lectrice le dernier monstre de l'histoire. Ce désir délirant, qui repose sur le fantasme d'une action de la littérature sur le réel commenté plus haut, peut se donner à lire par l'inscription de moments performatifs dans la narration. Sans entrer dans les débats philosophiques entourant la notion de performativité ${ }^{16}$, je me contenterai de la formulation proposée par Judith Butler, qui stipule que «dans la théorie des actes de discours, le performatif est cette pratique discursive qui réalise et produit ce qu'elle nomme " en ajoutant à cette définition une dimension répétitive, puisque "la performativité [...] est toujours la réitération d'une norme ou d'un ensemble de normes " (Butler 2009: 27). J'ai déjà mentionné les nombreux passages métanarratifs présents dans le texte de Cooper ; lors de ces moments, il arrive qu'une dimension performative de la narration sur un lecteur toujours implicite surgisse. C'est le cas, par exemple, dans ce passage où le narrateur ordonne au lecteur implicite de faire aller son imagination. Que le lecteur ou la lectrice véritable obéisse effectivement à cette suggestion importe peu, puisque c'est le désir du texte, son action fantasmée sur son lecteur modèle qui me paraissent signifiants. Vers le milieu du roman, on peut lire :

Supposons que la pièce où vous êtes en train de lire ceci ressemble aux
vestibules qui entrelacent mon récit. En d'autres termes, elle est flanquée
d'une pièce secrète ou d'un passage. [...] Contrairement à ce qu'indique la
notice biographique sur la couverture de ce livre ou ce fichier téléchargé, en
vérité je ne suis pas, oh, si j'essaie de deviner, mort ou emprisonné de longue
date. Non, je suis toujours dans la nature et en train d'écrire ce texte,
enfermé derrière le mur que vous regardez avec tant de confiance, mon œil
droit vous observant à travers un judas, utilisant votre visage absorbé,
méfiant ou excité pour ajuster mon récit. $(F M, 127)$

35 En utilisant un "supposons » qui unit le lecteur implicite et l'énonciateur sous un même «nous » afin de mieux le « diriger ", comme s'il le prenait par la main, le texte imite ici la structure pornotopique. Le performatif fantasmé du texte viserait ainsi à faire "apparaître", par la description et par l'utilisation de verbes à l'impératif seulement, dans l'esprit du "lecteur» l'espace du récit. Le narrateur, en somme, «brise » le quatrième mur en suggérant que le lecteur ou la lectrice se trouve dans le même espace que lui, et que - si nous nous identifions à la position du lecteur implicite - nous nous trouvons peut-être, au moment de lire, dans une pièce rappelant l'architecture à double fond du Château Étage. Le lecteur implicite se voit ainsi performativement "placé » dans la pornotopie par le biais d'une stratégie discursive dans laquelle le narrateur tente de se matérialiser à la manière d'un mauvais esprit 
dans l'espace « hors du livre ». Les doutes que le texte instaure sur la véracité du récit du narrateur soutiennent par ailleurs un autre moment performatif fantasmatique. À la fin du livre, toutes les données que nous avons sur le narrateur, sur l'histoire racontée et sur les lieux sont, suggère-t-on, potentiellement fausses, " au mieux, des analogies " $(F M, 82)$. Si vous me regardiez, dit le narrateur, vous «marmonneriez que je ne ressemble pas, mais alors pas du tout à ce que vous aviez imaginé », vous verriez aussi que l'endroit où je me trouve "n'est pas vraiment un loft, mais plutôt une mansarde " et que "la vue n'a rien de parisienne» (FM, 194). Il en va ainsi de presque tous les éléments du récit, présentés tantôt comme des versions (peut-être) jouées d'autres éléments diégétiques, dont l'existence est tantôt niée avant que cette négation ellemême soit niée. Impossible, à la fin du roman, de s'arrêter sur une version du récit plutôt qu'une autre. Le texte comme le narrateur, de toute manière, « ne croi[t] pas en l'honnêteté ou en une ineptie si complète qu'elle efface tous les mensonges » (FM, 51). Pour comprendre Le fol mabre, il faudrait ainsi accepter ce pacte de lecture où tout « est potentiellement crypté » $(F M, 82)$, où « la vérité est un détail technique » $(F M, 131)$ et où, à la manière de l'inconscient freudien qui ne connait pas l'opposition, « vous n'avez pas à craindre de mensonges ou de vérités contradictoires » (FM, 195).

36 Les romans qui se terminent par « ce n'était qu'un rêve » sont généralement frustrants et décevants en raison de la facilité d'une telle chute, qui dénie la fiction que tant de pages se sont efforcées de construire. Bien que ce ne soit pas la voie choisie par Cooper, qui suggère cette possibilité tout en laissant planer le doute sur celle-ci (comme sur les autres), j'aimerais en terminant essayer de penser ce qu'une telle fin peut signifier quant à la figure du monstre. Un autre moment performatif du récit me permet de proposer une piste d'interprétation, puisqu'elle montre que le texte vise, par ce brouillage, à faire du lecteur ou de la lectrice implicites le monstre ultime, peut-être le seul du récit. Vers la fin du roman, le narrateur annonce : «Si j’ai progressé comme je le souhaite, vous avez supporté les pages inflexibles de mon récit en sachant que je résoudrais les mystères qu'elles n'arrêtent pas de délimiter, mais vous commencez à vous douter que, pendant tout ce temps passé à animer mes personnages et anecdotes, vous avez simplement lu ce que vous voulez lire» (FM, 195). Celui ou celle qui a véritablement voulu assister au viol d'Alfonse, à l'assassinat sauvage et au massacre du corps d'un petit garçon, c'est peut-être celui ou celle qui tient le livre, nous dit Le fol marbre. Le texte tente ainsi de faire tomber la dernière barrière entre le monstre et la normalité, qu'on a longtemps pensés en dichotomie, et nous révèle que le lecteur implicite a inévitablement un rôle à jouer dans la formation du monstrueux, et qu'il n'est pas à l'abri de cette abjection sur laquelle s'édifie son humanité. Cette proposition inquiétante ne met autrement dit personne à l'abri d'un devenir-monstre et tente de produire, sur le plan fantasmatique, implicite et interne au roman, un monstre en la personne de son «lecteur » ou de sa "lectrice», en puisant aux registres performatifs du littéraire qui, tout comme le fol marbre, auraient dit-on parfois le pouvoir d'agir sur le réel, de réaliser ce qui est dit en le disant.

Le fol marbre met en texte trois changements marquant un tournant important dans la conception du monstre dans la fiction et dans la société contemporaine. Le roman présente un monstre, d'abord, qui apparaît désormais aux rangs des puissants, qui tend vers le dominant puisqu'il est composé comme l'hyperbole des logiques économiques et raciales du système politique capitaliste actuel. Ce monstre déjoue les conceptions classiques du corps et de l'identité en mettant à l'épreuve les dualismes qui servent à 
établir le genre comme le résultat du sexe biologique; la représentation de comportements monstrueux, socialement inacceptables, vise de plus à faire dérailler la logique identitaire et assimilationniste du discours homosexuel orthodoxe, en associant celle-ci à tout ce qu'elle met à l'écart pour s'établir comme une catégorie stable d'intelligibilité de la sexualité. Ce processus révèle le caractère construit de toute norme, constat radical que Le fol marbre applique à la monstruosité elle-même. Le roman de Cooper montre en effet que la monstruosité n'est pas innée, mais acquise, et qu'elle peut être produite par des dispositifs technologiques générant cette subjectivité. Le fol marbre laisse ainsi planer une possibilité troublante : si le monstre est le fruit d'une fabrication, le lecteur ou la lectrice implicites, pris.e.s dans les mailles du fol marbre, pourraient bien constituer les « véritables » monstres de l'histoire, puisqu'il et elle sont la scène de sa représentation imaginaire. En ouvrant cette possibilité, le roman de Dennis Cooper réalise ce que Jack Halberstam avançait en écrivant que " monstrosity always unites monstrous form with monstrous meaning " (SS, 11). Celui ou celle qui a véritablement voulu assister au viol d'Alfonse, à l'assassinat sauvage et au massacre d'un petit garçon, c'est peut-être celui ou celle qui tient le livre, aimerait nous dire Cooper. Il faudrait éventuellement commenter la récurrence de cette idée dans son œuvre, mais comme souvent chez lui, Le fol marbre affirme que l'ultime monstre, c'est le lecteur ou la lectrice, « mon semblable, mon frère [ou ma sœur] ».

\section{BIBLIOGRAPHIE}

La Bible. 2001 [ $\mathrm{I}^{\mathrm{er}}$ siècle de notre ère]. Paris et Montréal : Bayard et Médiaspaul.

BELLEMARE, Pierre, \& NAHMIAS, Jean-François. 2004. Crimes dans la soie. Paris : Albin Michel. BOURCIER, Sam. 2011. Queer Zones. Politiques des identités sexuelles et des savoirs. Paris : Éditions Amsterdam.

BRAIDOTTI, Rosi. 2013. The Posthuman. New York: Polity Press.

BUTLER, Judith. 2009 [1993]. Ces corps qui comptent. De la matérialité et des limites discursives du « sexe »(Charlotte Nordmann, trad.). Paris : Éditions Amsterdam.

BUTLER, Judith. 2005 [1990]. Trouble dans le genre. Le féminisme et la subversion de l'identité (Cynthia Kraus, trad.). Paris : La Découverte.

CHASSAY, Jean-François. 2014. Le monstre au bistouri. Neuilly-lès-Dijon : Le murmure.

COOPER, Dennis. 2016. Le fol marbre (Elsa Boyer, trad.). Paris : P.O.L.

COOPER, Dennis. 2011. The Marbled Swarm. New York: Harper Perennial.

COOPER, Dennis. 2004. Violence, faits divers, littérature (Bernard Hoepffner, trad.). Paris : P.O.L.

DENAULT, Alain. 2015. La médiocratie. Montréal : Lux, coll. « Lettres libres ».

FAUSTO-STERLING, Anne. 2012 [2000]. Corps en tous genres. La dualité des sexes à l'épreuve de la science (Oristelle Bonis \& François Bouillot, trad.). Paris : La Découverte. 
FÉREZ MORA, Pedro Antonio. 2014. “Dennis Cooper's The Sluts: Prosthetic and Performative (Homo)Sexuality” Revista de Filología Inglesa 35 : 71-88.

FÉREZ, MORA, Pedro Antonio. 2013. "Violence, Death, Sex and Psychoanalysis in Dennis Cooper's The Dream Police" Atlantis 35 (2) : 81-98.

HALBERSTAM, Jack. 1995. Skin Shows: Gothic Horror and the Technology of Monsters. Durhman and London: Duke University Press.

ISER, Wolfgang. 1985 [1972]. L'acte de lecture : théorie de l'effet esthétique (Evelyne Sznycer, trad.). Bruxelles : Pierre Mardaga.

JOOS, Jean-Ernest. 1999. « La fin du sexuel. Expérience, expérimentation et pratiques sexuelles dans les fictions de Dennis Cooper » Protée 27 (3) : 93-102.

KRISTEVA, Julia. 1980. Pouvoirs de l'horreur. Paris : Seuil.

LAQUEUR, Thomas. 2013 [1992]. La Fabrique du sexe. Essai sur le corps et le genre en Occident (Michel Gautier, trad.). Paris : Gallimard.

LEV, Leora (dir.). Enter at your own risk: the dangerous art of Dennis Cooper. Carnbury: Associated University Press.

MORNET, Daniel. 1929. Histoire de la clarté française, ses origines, son évolution, sa valeur. Paris : Payot.

PATOINE, Pierre-Louis. 2015. Corps/texte : pour une théorie de la lecture empathique (Cooper, Danielewski, Frey, Palahniuk). Lyon : ENS.

PICOCHE, Jacqueline. 2008. Dictionnaire étymologique du français. Paris : Le Robert.

PIKETTY, Thomas. 2013. Le Capital au XXI ${ }^{e}$ siècle. Paris : Seuil.

PRECIADO, Paul B. 2000. Manifeste contra-sexuel (Sam Bourcier, trad.). Paris : Balland.

PRECIADO, Paul B. 2011. Pornotopie : Playboy et l'invention de la sexualité multimédia (Serge Mestre, trad.). Paris : Climats.

PRECIADO, Paul B. 2014 [2008]. Testo Junkie : sexe, drogue et biopolitique (Paul B. Preciado, trad.). Paris : J'ai Lu.

STIÉNON, Valérie et Érika WICKY. 2013. « Un siècle de physiognomonie » Études françaises, 49 (3) : $5-14$.

SWIGGERS, Pierre. 1987. «À l'ombre de la clarté française » Langue française $75:$ 5-21.

WITTIG, Monique. 2013. La pensée straight. Paris : Éditions Amsterdam.

\section{ANNEXES}

\section{Résumé du roman}

Le fol marbre s'ouvre sur la visite du narrateur parisien d'un château en province, le "Château Étage », hanté selon les journaux depuis la mort d'un jeune garçon emo, Claude, retrouvé noyé dans la rivière. Le protagoniste rencontre les membres de la famille qui habite le château, en pince pour le frère du garçon mort, Serge, qu'il enfermera bientôt dans le coffre de sa voiture avec l'assentiment du père, qui confesse avoir déjà commis sur Serge nombre viols en plus d'une tentative de meurtre. Avant de quitter le château, sur lequel le narrateur a conclu une entente d'achat avec le père, ce 
dernier lui fait visiter un réseau complexe de passages secrets qui structurent la maison, des couloirs qui permettent d'accéder à toutes les pièces afin de surveiller ce qui s'y déroule. Le narrateur et son chauffeur reprennent la route vers Paris avec Serge à l'arrière. Le deuxième chapitre se clôt sur un passage métanarratif, au cours duquel le narrateur révèle que tous les éléments de l'histoire que nous avons lue jusqu'à présent sont « potentiellement crypté[s] », qu'ils sont « au mieux des analogies » $(F M, 82)$.

Dans les chapitres suivants, on quitte la trame principale pour en apprendre davantage sur les origines du narrateur. Fils d'un père milliardaire, il a grandi dans une maison immense qui rappelle le Château Étage en ce qu'elle est aussi charpentée par des corridors secrets. Le narrateur a un jeune frère, Alfonse, garçon amateur de mangas et de blogs de discussion en ligne, membre d'une communauté, les Flatsos, des « accros à tout ce qui est écrasé » $(F M, 88)$ dont le fantasme est de devenir aussi minces qu'une feuille de papier. La réalisation de ce désir constitue la partie la plus horrible du roman. Le meurtre d'Alfonse par François et Azmir, respectivement l'ami et le chauffeur de leur défunt père, et tous les sévices sexuels qui l'accompagnent nous sont décrits par le narrateur qui observe la scène, puis qui participe à l'aplatissement du cadavre du garçon à l'aide d'un baril.

Dans le chapitre suivant, le narrateur apprend, grâce au testament de son père, que ce dernier aurait fait bâtir de son vivant un " petit bidule massif et surnaturel » (FM, 225) abritant un théâtre, en province. Sa maison parisienne, quant à elle, a été rachetée par François, auquel le narrateur rend visite. Il rencontre ainsi son fils, Didier, à qui ils décident tous deux de faire subir des chirurgies plastiques pour rapprocher son visage de celui d'Alfonse. Ce chapitre approfondit le doute semé au début du livre quant à l'histoire qui nous est racontée. Au détour d'un paragraphe, le narrateur laisse planer l'idée que tout son récit serait pure fantasmagorie. Se trouve-t-il vraiment à Paris ? Estil le bellâtre cannibale qu'il prétend être ? Les passages secrets existent-ils ? La fin du roman, qui éclaircit autant qu'elle obscurcit le mystère planant autour du théâtre que son père aurait fait construire, ne résout rien, mais ajoute une autre couche à ce feuilleté de faux-semblants que monte, puis démolit sans cesse la parole envoûtante de celui qui nous le raconte.

\section{NOTES}

1. À l'adresse https://denniscooperblog.com/

2. COOPER, Dennis. 2016. Le fol marbre, traduction d'Elsa Boyer. Paris: P.O.L, p. 82. Désormais abrégé par $F M$ et cité entre parenthèses dans le corps du texte. La première personne du singulier dans les passages cités n'est jamais modifiée par souci de ton et parce que les guillemets suffisent à marquer le changement de narrateur dans la phrase. À l'occasion, j'ai inséré dans mes citations des passages de la version originale anglaise jugés éclairants : COOPER, Dennis. 2011. The Marbled Swarm. Harper Perennial: New York. Un résumé de la diégèse est disponible en annexe.

3. Jack Halberstam analyse en ce sens les romans victoriens Dracula et Frankenstein. Voir HALBERSTAM, Jack. 1995. Skin Shows: Gothic Horror and the Technology of Monsters. Durhman and London: Duke University Press, p. 22. Désormais abrégé par SS et cité entre parenthèses dans le corps du texte.

4. «Gothic monsters, furthermore, differ from the monsters that come before the nineteenth century in that the monsters of modernity are characterized by their proximity to humans » (SS, 23). 
5. Ce qui ne veut évidemment pas dire que la figure du «beau monstre " est nouvelle. Les exemples abondent sur nos écrans (pensons à la "vamp ", aux sorcières de Suspiria [2018] ou aux séduisants vampires contemporains) ou dans la littérature (Le portrait de Dorian Gray, American Psycho). Comme nous le verrons, ils se généraliseraient selon Jack Halberstam à l'époque récente parce que le monstre, dans l'imaginaire contemporain, serait plus fréquemment construit à partir de traits appartenant au «dominant» qu'au «marginal». Sur les rapports physiognomoniques entre monstruosité et aspect physique, voir ses analyses de Frankenstein et de Dracula (SS, 28-85). Son analyse du Silence des agneaux (SS, 161-177) perçoit quant à elle chez le personnage de Buffalo Bill une figure de « beau monstre ».

6. J'emploie ici le concept d'Iser qui désigne une «structure du lecteur inscrite dans le texte », un «modèle transcendantal qui permet d'expliquer comment le texte de fiction produit un effet et acquiert un sens » (Iser 1985 : 70). Lorsque je fais référence à celui ou celle qui lit le texte dans la réalité, j'utilise « lecteur ou lectrice " plutôt que « lecteur implicite », que je conserve comme tel de la traduction de L'acte de lecture, faute de neutre en langue française.

7. À la même page, Piketty donne Liliane Bettencourt comme exemple d'héritière. Dans Le fol marbre, le narrateur souligne quant à lui « qu'une personne du monde de l'art parisien [a] versé un pot-de-vin pour acheter aux poussières de [son] père un emplacement luxueux dans le cimetière Montparnasse qui avait été conservé pour Liliane Bettencourt avant ses scandales » (FM, 166).

8. Crimes dans la soie de Pierre Bellemare et Jean-François Nahmias en fournit un florilège d'exemples.

9. Le roman de Sade qui a inspiré le film de Pasolini est d'ailleurs une influence majeure pour l'écrivain américain (Cooper 2004).

10. Dans la traduction française d'Elsa Boyer, le narrateur s'adresse à son allocutaire - le lecteur implicite - tantôt avec le pronom «tu », tantôt avec le pronom « vous », et ce, sans qu'on puisse identifier un destinataire différent dans un cas ou l'autre.

11. Fait de prendre plaisir à imaginer être avalé.e ou avaler le/la partenaire.

12. Contrairement, par exemple, à d'autres pratiques sexuelles scriptées, comme le S\&M ou le BDSM. Chez Cooper, aucune pratique ne semble résoudre la dispersion fragmentaire des actes.

13. Pedro Antonio Férez Mora, dans un texte brillant, analyse sous le signe de la " prothèse » un déplacement analogue de la sexualité dans le roman The Sluts (Férez Mora 2014 : 74-78).

14. Sur la notion de posthumain, voir Braidotti 2013.

15. Le Château Étage est d'ailleurs, au début et à la fin du roman, présenté comme un théâtre (FM, $53 ; 220)$.

16. Notamment entre Jacques Derrida et John Searle.

\section{RÉSUMÉS}

Cet article fait appel à la théorie queer (Butler, Preciado, Halberstam) pour proposer une analyse du Fol marbre de Dennis Cooper, en poursuivant l'hypothèse que le roman vise à révéler les mécanismes d'édification des normes identitaires et sexuelles. Pour ce faire, je montre que le roman de Cooper questionne le lien entre le genre et le monstre : le monstre, à la manière du genre, est dans Le fol marbre le fruit d'une fabrication culturelle et discursive, fabrication que le texte nous permet de saisir comme telle, en explorant les différents dispositifs sexuels et 
culturels qui la produisent. Le monstre qui narre Le fol marbre est construit à l'intersection de caractéristiques économiques, raciales et criminelles, de comportements ou de désirs sexuels frappés d'un interdit social qui servent à composer son abjection fictive. Le roman coalise ainsi certains changements importants dans l'imaginaire contemporain du monstre, dont l'histoire est esquissée par Jack Halberstam dans Skin Shows: Gothic Horror and the Technology of Monsters. Les incarnations contemporaines du monstre dans la fiction marqueraient, selon Halberstam, un changement quant à la fonction sociale de la figure. Plutôt que d'unir, comme à l'époque victorienne, sous son masque grimaçant différents éléments qui menacent la culture dominante afin de protéger celle-ci en identifiant le monstre comme son envers, son extérieur, son Autre absolu, le monstre contemporain se caractériserait par son rapprochement, sa proximité avec l'humain et avec la norme. Le narrateur/monstre du Fol marbre fonctionne comme un instrument narratif servant à critiquer la culture dominante plutôt qu'à en affermir les assises. Le monstre lui-même ainsi que les actes immondes qu'il commet sont rendus possibles en raison de la marginalité ultra-permissive dans laquelle se trouve ce personnage ultra-riche. Dans le Fol marbre, c'est un dérapage du monde social qui produit le monstrueux, et non l'inverse. Ce passage du monstre vers le dominant s'accompagne d'actions et de désirs exorbitants, connotés comme abjects dans le récit et qui visent à ébranler la notion classique du sujet, et par là même un ensemble de binarismes qui fondent son projet. Le fol marbre, plutôt que de proposer un modèle stable du monstrueux, lui substitue une conception productive, générative s'apparentant à la conception butlérienne du genre. Le monstre chez Cooper est le fruit d'un processus de fabrication qui l'institue comme tel à la fois dans la diégèse et dans le texte. Par le fait même, le roman menace les limites autrefois stables du monstrueux : si on ne naît pas monstre, on le devient, personne n'est à l'abri de ce devenir-monstre qui hante Le fol marbre. Pas même le lecteur ou la lectrice.

This article uses queer theory (Butler, Preciado, Halberstam) to analyse Dennis Cooper's The Marble Swarm, and shows how that the novel reveals the mechanisms of edification of sexual norms. In fact, Cooper's novel questions the link between gender and monster: the monster, just as gender, is exhibited as a cultural and discursive production, through the exploration of the sexual and cultural devices that produce it. The monster who narrates the novel is built at the intersection of socially prohibited economic, racial and criminal behaviors and sexual desires, which compose his fictional abjection. The novel therefore testifies to some important changes in the contemporary imagination surrounding the monster. According to Halberstam in Skin Shows: Gothic Horror and the Technology of Monsters, contemporary fictional incarnations of monsters herald a change in the representation of the figure. When the Victorian monster embodied everything that threatened the dominant culture in order to protect it, the contemporary monster is characterized by its proximity to the human and to the norm. Thus, the narrator/ monster of The Marble Swarm functions as a narrative instrument that criticises dominant culture rather than strengthening it. The monster and its actions are made possible thanks to the overpermissive, marginal space the wealthy narrator was born into. The Marble Swarm rather than offering a stable model of the monstrous coins a generative conception of it similar to the Butlerian concept of gender. Cooper's monster is the result of a manufacturing process that establishes it as such in both the diegesis and the text. Through the same strategy, the novel threatens the once stable limits of the monstrous: one is not born but rather becomes a monster, and no one is safe from this becoming-monster that haunts The Marble Swarm. Not even the reader. 
INDEX

Thèmes : Recherches

Keywords : queer studies, monster, gender and sexuality, contemporary literature, American literature, Dennis Cooper, homosexuality, contemporary novel

Mots-clés : études queer, monstre, genre et sexualité, littérature contemporaine, littérature américaine, Dennis Cooper, homosexualité, roman contemporain

\section{AUTEUR}

\section{KEVIN LAMBERT}

Kevin Lambert est écrivain et doctorant en Littératures de langue française (Université de Montréal). Il a publié deux romans, Tu aimeras ce que tu as tué (Héliotrope 2017) et Querelle de Roberval (Héliotrope 2018 ; Querelle, Le Nouvel Attila, 2019), des essais et des nouvelles. Ses travaux portent sur la théorie queer, les théories de la création littéraire et l'histoire du livre (Hélène Cixous, Victor-Lévy Beaulieu, Marie-Claire Blais, Stéphane Mallarmé, Lautréamont). 